International Mathematical Forum, 2, 2007, no. 27, 1319 - 1328

\title{
On Mathematical Foundations of the Plausibility Theory
}

\author{
J. M. Sigarreta \\ Department of Mathematics \\ University Carlos III of Madrid, Spain \\ josemaria.sigarreta@uc3m.es \\ P. Ruesga \\ Department of Specific Didactic \\ University of Burgos, Spain \\ pruesga@ubu.es \\ M. Rodriguez \\ Department of Mathematics, U.A.H \\ mrodriguez@yahoo.com
}

\begin{abstract}
This article constructs a theoretical framework enabling the logic of plausibility to be axiomatised and for decisions about plausibility to be automated. One result is that undecidability presented in the initial theory is resolved. To arrive at a logical-mathematical basis of the theory of plausibility an axiomatization of plausibility logic based on notions of evidence is proposed. It is through these axioms that this logic is characterized and its properties deduced.
\end{abstract}

Mathematics Subject Classification: 68R01; 03B70

Keywords: Computational logic, formal logic, logic of plausibility

\section{Introduction}

The theory of plausibility is being successfully applied in different scientific fields such as Economics, Sociology and Computing Sciences. In this latter 
discipline, it is used in the implementation of computational and/or collaborative tools and in the definition of computational designs (see [3], [5], [6], [4] and $[1])$.

These works present a theory of plausibility for computer architecture and design, with a view to the formalization of such designs from a computational standpoint. More specifically, in [1] is proposed a theory to explain the plausibility of designs that consist of principles that characterize their own nature. They define ways of formulating statements based on plausibility logic in order to arrive at propositions regarding the merits and shortcomings of computational design which serve as guidelines for reasoning in the development of such designs. In [5], an evolutionary model of computing design processes is also described. In this work the plausibility of a design is dependent on its history. In other words, it is dependent on (a) the sequence of steps associated with decisions on design, (b) the underlying cause-effect of the relations between design decisions, and (c) on the nature of the evidence used by the designer to justify such decisions and/or to establish the basic characteristics of the design that will satisfy a particular set of requirements. The approximation was used to design a special multiprocessor to serve as a computer architecture simulator (CASE).

The theory of plausibility explains the acceptability of a decision. In general, a decision is a non-empty and finite set of statements, called restrictions, which specify their relevant attributes. In determining the plausibility of a decision, the logic that is relied on at present is developed at a primary level and is completely intuitive-experimental. The descriptive predominates over the formal, which makes it difficult to identify essential aspects. This situation complicates the understanding and application of the theory. Up until the present, plausibility logic has not been able to rely on a deductive system that is able to characterize its connectives in terms of plausibility, since as shown in citeaugu, it is difficult to calculate the plausibility of a decision in terms of evidence, and there are even problems of decidability. Currently, plausibility logic cannot be used to deduce other logical properties, which might be of interest in the application of the theory, including those which might offer insight into the nature and the scope of plausibility logic.

In this study, the Theory of Plausibility is formalized at a wider level so as to explain decision-making processes in mathematical terms. A functional and numerical definition of both evidence and plausibility is given, from which is developed a multivalent (pentavalent) logic of plausibility, which has not been contemplated in the previously mentioned works. On the basis of these mathematical elements, the axiomatization and development of the logic of plausibility is described, which represents the fundamental aspect of this research. For the first time, axioms are formulated that constitute functional evidence, quantificational evidence and variability between evidence and plau- 
sibility. This axiomatic system serves as the basis for a more rigorous development of the essential elements of the logic.

\section{Preliminares Notes}

Plausibility of a decision is determined by the acceptability of a number which is associated with statements which relate to such a decision. In general, these statements describe relevant characteristics of the decision.

The plausibility of a statement $\mathrm{A}$ is defined in terms of evidence as to the truthfulness of $\mathrm{A}$, with reference to the following five states of plausibility: unsatisfactory, doubtful, undetermined, feasible/possible and satisfactory, represented by $0,1,2,3$ and 4 , respectively.

Different methods exist, which are precise and formal, approximative and experimental, to evaluate how strong the evidence is that statement $\mathrm{A}$ is true. The approach to plausibility through evidence adds great flexibility to the theory, and allows adjustment to problems which are of differing characters. In many cases, this is what is needed to arrive at a satisfactory solution, as for example, when one needs to use approximate reasoning (diffuse logic) instead of a more precise method of solution.

In plausibility theory, due to the evolutive nature of decisions that are successively refined or reconsidered, the evidence is subject to change, and, as a result, so are the states of plausibility. In order to resolve this problem, plausibility theory breaks down the decision into stages, in such a way that there is no possibility of changes in the evidence.

Therefore, in the process of taking the decision, the evidence depends on the stage and the statement under consideration. This gives rise to functional representations of evidence to the contrary $(e c)$, evidence in favour $(e f)$, significant evidence to the contrary $(s e c)$ and significant evidence in favour (sef) using the following numerical functions. Let $\mathrm{K}$ be the set of stages and $\mathrm{F}$ the set of statements, then

Definition 2.1. ec, ef: $K \times F \longrightarrow\{0,0.5,1\}$ and sec, sef: $K \times F \longrightarrow$ $\{0,1\}$ such that,

$e c(k, A)= \begin{cases}1 & \text { if it is known that evidence to the contrary exists; } \\ 0.5 & \text { if it is not known whether evidence in contrary exists or not; } \\ 0 & \text { if it is known that no evidence to the contrary exists. }\end{cases}$

$e f(k, A)= \begin{cases}1 & \text { if it is known that evidence in favour exists; } \\ 0.5 & \text { if it is not known whether evidence in favour exists or not; } \\ 0 & \text { if it is known that no evidence in favour exists. }\end{cases}$ 
$\sec (k, A)= \begin{cases}1 & \text { if ec }(\mathrm{k}, \mathrm{A})=1 \text { and the evidence is known to be significant; } \\ 0 & \text { other cases. }\end{cases}$

$\operatorname{sef}(k, A)= \begin{cases}1 & \text { if } \text { ef }(\mathrm{k}, \mathrm{A})=1 \text { and the evidence is known to be significant; } \\ 0 & \text { other cases. }\end{cases}$

Based on this definition, the plausibility of a statement is expressed through the function qualify, represented by qual, where:

Definition 2.2. qual: $K \times F \longrightarrow\{0,1,2,3,4\}$ such that,

$$
\operatorname{qual}(k, A)= \begin{cases}0 & \text { if } \operatorname{ec}(\mathrm{k}, \mathrm{A})=1 \text { and } \sec (\mathrm{k}, \mathrm{A})=1 \\ 1 & \text { if } \mathrm{ec}(\mathrm{k}, \mathrm{A})=1 \text { and } \sec (\mathrm{k}, \mathrm{A})=0 \\ 2 & \text { if } \mathrm{ec}(\mathrm{k}, \mathrm{A})=0.5 \\ 3 & \text { if } \mathrm{ec}(\mathrm{k}, \mathrm{A})=0 \text { and } \operatorname{sef}(\mathrm{k}, \mathrm{A})=0 \\ 4 & \text { if } \operatorname{ec}(\mathrm{k}, \mathrm{A})=0 \text { and } \operatorname{sef}(\mathrm{k}, \mathrm{A})=1\end{cases}
$$

As qual is a function of a given stage, each statement takes one and only one of the five states of plausibility, which is fundamental for plausibility logic to be a multivalent logic.

Remark 1. If ec $(k, A)=\operatorname{ec}(k, B), \sec (k, A)=\sec (k, B), \operatorname{sef}(k, A)=\operatorname{sef}(k, B)$ then $\operatorname{qual}(k, A)=\operatorname{qual}(k, B)$.

\section{Main Results}

Theorem 3.1. The logic of plausibility is undecidable.

Proof. It is sufficient to prove that $q u a l(k, A)=0$ does not determine qual $(k, \backsim$ $A)$. In [2] and [4], only the following properties are referred to as functional evidence of the connective $\backsim$ : ef $(k, \backsim A)=e c(k, A), \sec (k, \backsim A)=\operatorname{sef}(k, A)$ and $\operatorname{sef}(k, \backsim A)=\sec (k, A)$.

By Definition 2.2, $\operatorname{qual}(k, A)=0$ if and only if,

- $e c(k, A)=1$ if and only if $e f(k, \backsim A)=1$

- $\sec (k, A)=1$ if and only if $\operatorname{sef}(k, \backsim A)=1$.

Hence, $\operatorname{qual}(k, A)=0$ if and only if $\operatorname{ef}(k, \sim A)=\operatorname{sef}(k, \sim A)=1$. By Definition 2.2 , ef $(k, \backsim A)=1$ and $\operatorname{sef}(k, \backsim A)=1$ is not sufficient to determine qual $(k, \backsim A)$. 
Plausibility regions and axiomatization of the logic of plausibility.

Evidence to the contrary is determinative for the state of plausibility of a statement, which leads us to classify plausibility by regions.

Theorem 3.2. The relation: $(a, b) \in R$ if and only if ec $(k, A)=e c(k, B)$ such that qual $(k, A)=a$ and qual $(k, B)=b$ is an equivalence relation on $P=\{0,1,2,3,4\}$.

Proof. $R$ is reflexive on $P$ : Let a $P$, then $e c(k, A)=e c(k, A)$ such that qual $(k, A)=a$. Thus $(a, a) \in R . \quad R$ is symmetrical: Let $(a, b) \in R$, then $e c(k, A)=e c(k, B)$ such that qual $(k, A)=a$ and qual $(k, B)=b, e c(k, B)=$ $e c(k, A)$ such that qual $(k, B)=b$ and qual $(k, A)=a$. Hence $(b, a) \in R$. $R$ is transitive: Let $(a, b),(b, c) \in R$, then $e c(k, A)=e c(k, B)$ and $e c(k, B)=$ $e c(k, C)$ such that $q u a l(k, A)=a, \operatorname{qual}(k, B)=b$ and $q u a l(k, C)=c$. So $q u a l(k, A)=\operatorname{qual}(k, B)$. But $e c(k, A)=e c(k, C)$ such that qual $(k, A)=a$ and qual $(k, C)=c$. Hence $(a, c) \in R$.

Because $R$ is an equivalence relation on $P$, it determines a partition on the latter set $P, P / R=\{\{0,1\},\{2\},\{3,4\}\}$.

Definition 3.3. $R_{0}=\{0,1\}, R_{1}=\{2\}$ and $R_{2}=\{3,4\}$, in which $R_{0}$ is rejection, $R_{1}$ indetermination and $R_{2}$ acceptance.

The logic of plausibility is a semantic-extensional method of a multivalent propositional logic (pentavalent: five states of plausibility) and it is non monotonic in nature. Its purpose is to determine functional plausibility. It is characterized by five connectives ( $p$-connectives): $\sim, \wedge, \vee, \Longrightarrow, \Longleftrightarrow$ known as negation, conjunction, disjunction, implication and coimplication. These latter connectives determine both the evidence and the plausibility and the statements which are not atomic.

The principles and properties that appear at an intuitive level in all works are taken as the base on which to establish the axioms that uphold the logic of plausibility. The axioms consist of an appropriate selection of the latter principles or properties, in such a way that they encapsulate the minimum required to deduce the intuitively desirable properties of plausibility logic, essentially a characterization of the connectives in terms of plausibility.

If the evidence for a statement is functional, the value of such evidence will be determined from the axioms or the properties that are derived from them. The axioms proposed in this paper for the logic of plausibility have not been published before.

From the seven axioms presented A1, A2, A3, A4 and A6 figure among the principles that explicitly or implicitly appear in [1] and [2]. In addition, two axioms are included A5 and A7.

The $A 7$ is intuitive, which is to say, if $\operatorname{qual}(k, A)=\operatorname{qual}(k, B)$ it is to be expected that $\operatorname{se} f(k, A)=\operatorname{sef}(k, B)$. Although less is demanded by axiom 
$A 7$, in itself it is a much weaker condition, as it only considers the case of $q u a l(k, B)=3$. The $A 5$ is deduced from the need to characterize the connective $\vee$.

$$
\begin{aligned}
& \operatorname{Axiom}(A 1)\left\{\begin{array}{l}
\sec (k, \backsim A)=\operatorname{sef}(k, A) ; \\
\operatorname{sef}(k, \backsim A)=\sec (k, A) ; \\
\operatorname{ec}(k, \backsim A)=1-\operatorname{ec}(k, A) .
\end{array}\right. \\
& \text { Axiom }(A 2)\left\{\begin{array}{l}
\operatorname{ec}(k, A \wedge B)=\max (\operatorname{ec}(k, A), \operatorname{ec}(k, B)) \\
\sec (k, A \wedge B)=\max (\sec (k, A), \sec (k, B)) \\
\operatorname{sef}(k, A \wedge B)=\min (\operatorname{sef}(k, A), \operatorname{sef}(k, B)) .
\end{array}\right. \\
& \text { Axiom }(A 3)\left\{\begin{array}{l}
\operatorname{ec}(k, A \vee B)=e c(k, \backsim(\backsim A \wedge \backsim B)) ; \\
\sec (k, A \vee B)=\sec (k,(\backsim A \wedge \backsim B)) ; \\
\operatorname{sef}(k, A \vee B)=\operatorname{sef}(k, \backsim(\backsim A \wedge \backsim B)) .
\end{array}\right. \\
& \text { Axiom }(A 4)\left\{\begin{array}{l}
\operatorname{ec}(k, A \Longrightarrow B)=e c(k, \backsim A \vee B) \\
\sec (k, A \Longrightarrow B)=\sec (k, \backsim A \vee B) ; \\
\operatorname{sef}(k, A \Longrightarrow B)=\operatorname{sef}(k, \backsim A \vee B)
\end{array}\right. \\
& \text { Axiom }(A 5)\left\{\begin{array}{l}
\operatorname{ec}(k, A \Longleftrightarrow B)=\operatorname{ec}(k,(A \Longrightarrow B) \wedge(B \Longrightarrow A)) ; \\
\sec (k, A \Longleftrightarrow B)=\sec (k,(A \Longrightarrow B) \wedge(B \Longrightarrow A) ; \\
\operatorname{sef}(k, A \Longleftrightarrow B)=\operatorname{sef}(k,(A \Longrightarrow B) \wedge(B \Longrightarrow A))
\end{array}\right. \\
& \text { Axiom }(A 6)\left\{\begin{array}{l}
\operatorname{ec}(k, \forall x A x)=\operatorname{ec}\left(k, A x_{1} \wedge A x_{2} \cdots \wedge A x_{n}\right) \\
\sec (k, \forall x A x)=\sec \left(k, A x_{1} \wedge A x_{2} \cdots \wedge A x_{n}\right) \\
\operatorname{sef}(k, \forall x A x)=\operatorname{sef}\left(k, A x_{1} \wedge A x_{2} \cdots \wedge A x_{n}\right) \\
\operatorname{ec}(k, \exists x A x)=\operatorname{ec}\left(k, A x_{1} \vee A x_{2} \cdots \vee A x_{n}\right) \\
\sec (k, \exists x A x)=\sec \left(k, A x_{1} \vee A x_{2} \cdots \vee A x_{n}\right) \\
\operatorname{sef}(k, \exists x A x)=\operatorname{sef}\left(k, A x_{1} \vee A x_{2} \cdots \vee A x_{n}\right)
\end{array}\right.
\end{aligned}
$$$$
\text { Axiom }(A 7)\{\operatorname{qual}(k, A)<\operatorname{qual}(k, B)=3 \text { then sef }(k, A) \leq \operatorname{sef}(k, B) \text {. }
$$

Properties of the logic of plausibility. The more general theorems are the only ones that are presented deductively, given that the rest may be demonstrated with the same techniques. The following theorem allows us to state that $e c(k, \backsim A)=1-e c(k, A)$ is equivalent to $\operatorname{qual}(k, A)$ and $\operatorname{qual}(k, \backsim A)$ being located in symmetrical regions of plausibility. In other words, whenever $\operatorname{qual}(k, A)$ is in a region of rejection, indetermination of acceptance, then $q u a l(k, \backsim A)$ is also in a region of acceptance, indetermination or rejection, which coincides with what occurs in practice. 
Theorem 3.4. ec $(k, \backsim A)=1-e c(k, A)$ if, and only if, qual $(k, A) \in R_{i}$ implies qual $(k, \backsim A) \in R_{2-i}$.

Proof. qual $(k, A) \in R i$. i $=0$. If $q u a l(k, A) \in R_{0}$, then qual $(k, A) \in\{0,1\}$, by Definition 3.3 and $e c(k, A)=1$ by Definition 2.2. By hypothesis $e c(k, \backsim A)=$ 0 . Therefore, according to Definition $2.2, \operatorname{qual}(k, \sim A) \in\{3,4\}$, that is, by Definition 3.3 qual $(k, \backsim A) \in R_{2}$. The proofs for $i=1,2$ are analogous. Thus, qual $(k, A) \in R_{i}$ implies qual $(k, \sim A) \in R_{2-i}$.

If $e c(k, A)=0$, by Definition 2.2, $\operatorname{qual}(k, A) \in\{3,4\}$ and by Definition $3.3, \operatorname{qual}(k, A) \in R_{2}$, as shown in the latter section qual $(k, \sim A) \in R_{0}$, by Definition 3.3, qual $(k, \backsim A) \in\{0,1\}$, and by Definition $2.2, e c(k, \backsim A)=1$. Which is to say: $e c(k, \backsim A)=1-e c(k, A)$.

If $e c(k, A)=0.5$, by Definition $2.2, \operatorname{qual}(k, A) \in\{2\}$ and by Definition 3.3, $\operatorname{qual}(k, A) \in R_{1}$, as shown in the latter section qual $(k, \sim A) \in R_{1}$, by Definition 3.3, qual $(k, \backsim A) \in\{2\}$, and by Definition 2.2, ec $(k, \backsim A)=0.5$. Which is to say: $e c(k, \backsim A)=1-e c(k, A)$. The analogous way we proof the same relation for $e c(k, A)=1$. By combining both part we arrive at the general result.

Notice that

Lemma 3.5. If $a, b$ and $c$, are real numbers, then $a-\max (a-b, a-c)=$ $\min (b, c)$.

Theorem 3.6.
i) $e c(k, A \vee B)=\min (e c(k, A), e c(k, B))$;
ii) $\sec (k, A \vee B)=\min (\sec (k, A), \sec (k, B))$;
iii) $\operatorname{sef}(k, A \vee B)=\max (\operatorname{sef}(k, A), \operatorname{sef}(k, B))$.

Proof. Item $i)$ making $a=1, b=e c(k, A)$ and $c=e c(k, B)$ in Lemma 3.5 and using Axiom 1; Axiom 2 and Axiom 3 follow the result. The analogous way we obtained the other results.

\section{Theorem 3.7.}

a) $e c(k, A \Longrightarrow B)=\min (1-e c(k, A), e c(k, B))$;

b) $\sec (k, A \Longrightarrow B)=\min (1-\sec (k, A), \sec (k, B))$;

c) $\operatorname{sef}(k, A \Longrightarrow B)=\max (\sec (k, A), \operatorname{sef}(k, B))$;

d) $e c(k, A \Longleftrightarrow B)=\max (\min (1-e c(k, A), e c(k, B)), \min (e c(k, A), 1-$ $e c(k, B)))$; 
e) $\sec (k, A \Longleftrightarrow B)=$

$\max (\min (1-\sec (k, A), \sec (k, B)), \min (\sec (k, A), 1-\sec (k, B))) ;$

f) $\operatorname{sef}(k, A \Longleftrightarrow B)=$

$\min (\max (\sec (k, A), \operatorname{sef}(k, B)), \max (\operatorname{sef}(k, A), \sec (k, B)))$.

In the first place, we will prove items $a$ ) and $d$ ). The items $b$ ) and $c$ ) are proven by analogy with $a$ ) and items $e$ ) and $f$ ) by analogy with $d$ ).

Proof. Item a) by Axiom 4; Theorem 3.6 and Axiom 1. We have ec(k, $A \Longrightarrow$ $B)=e c(k, \backsim A \vee B)=\min (e c(k, \backsim A), e c(k, B))=\min (1-e c(k, A), e c(k, B))$.

Item $d$ ) by Axiom 5; Axiom 2 and item $a)$ we have $e c(k, A \Longrightarrow B)=$ $e c(k,(A \Longrightarrow B) \wedge(B \Longrightarrow A))=\max (e c(k, A \Longrightarrow B), e c(k, B \Longrightarrow A))=$ $\max (\min (1-e c(k, A), e c(k, B)), \min (1-e c(k, B), e c(k, A)))=\max (\min (1-$ $e c(k, A), e c(k, B)), \min (e c(k, A), 1-e c(k, B)))$.

Notice that

\section{Remark 2.}

- qual $(k, A)=$ qual $(k, B)$ implies ec $(k, B) \leq e c(k, A)$.

- ec $(k, B)=e c(k, A)$ implies $\sec (k, B) \leq \sec (k, A)$ or $\operatorname{sef}(k, B) \leq \operatorname{sef}(k, A)$.

Moreover, qual $(k, A)=\operatorname{qual}(k, B) \in\{3,4\}$ implies, sef $(k, A)=\operatorname{sef}(k, B)$. Another hand; using Theorem 3.6; Theorem 3.4; Lemma 3.5; Remark 1 and Axioms 1,2,3,4, and 5. We obtained the following result

Proposition 3.8.

a) $\operatorname{qual}(k, A \wedge B)=\operatorname{qual}(k, \backsim(\backsim A \vee \backsim B))$;

b) $q u a l(k, A \vee B)=\operatorname{qual}(k, \backsim(\backsim A \wedge \backsim B))$;

c) $\operatorname{qual}(k, A \Longrightarrow B)=\operatorname{qual}(k, \sim A \vee B)$;

d) $\operatorname{qual}(k, A \Longleftrightarrow B)=\operatorname{qual}(k,(A \Longrightarrow B) \wedge(B \Longrightarrow A))$.

The following theorem is the formulation and proof that characterizes and, in particular, enables automatization of functional plausibility and as a result the resolution of problems of undecidability.

\section{Theorem 3.9.}

a) $\operatorname{qual}(k, \sim A)=4-\operatorname{qual}(k, A)$;

b) $q u a l(k, A \wedge B)=\min (q u a l(k, A), q u a l(k, B))$; 
c) $\operatorname{qual}(k, A \vee B)=\max (q u a l(k, A), \operatorname{qual}(k, B))$;

d) $\operatorname{qual}(k, A \Longrightarrow B)=\max (4-\operatorname{qual}(k, A), \operatorname{qual}(k, B))$;

e) $\operatorname{qual}(k, A \Longleftrightarrow B)=$ $\min (\max (4-\operatorname{qual}(k, A), \operatorname{qual}(k, B)), \max (q u a l(k, A), 4-\operatorname{qual}(k, B)))$.

Proof. Item $a)$ Let qual $(k, A)=0$ by Definition $2.2, \operatorname{ec}(k, A)=\sec (k, A)=1$, by Axiom $1, e c(k, \backsim A)=0$ and $\operatorname{sef}(k, \sim A)=1$ by Definition 2.2, qual $(k, \backsim$ $A)=4$. Hence qual $(k, \sim A)=4-\operatorname{qual}(k, A)$. Let $q u a l(k, A)=1$ by Definition $2.2, \operatorname{ec}(k, A)=1$ and $\sec (k, A)=0$, by Axiom $1, \operatorname{ec}(k, \sim A)=0$ and $\operatorname{sef}(k, \backsim$ $A)=0$, by Definition 2.2, qual $(k, \backsim A)=3$. Hence qual $(k, \backsim A)=4-$ $q u a l(k, A)$. Using the same reasoning for $q u a l(k, A)=2 ; \operatorname{qual}(k, A)=3$ and $\operatorname{qual}(k, A)=4$ we have that $\operatorname{qual}(k, \sim A)=4-\operatorname{qual}(k, A)$.

Item $b)$

Let $q u a l(k, A) \leq q u a l(k, B)$, then

i.1) $\min (q u a l(k, A), \operatorname{qual}(k, B))=\operatorname{qual}(k, A)$.

i.2) By Remark $2, e c(k, B) \leq e c(k, A)$ and $\sec (k, B) \leq \sec (k, A)$.

Thus $\max (e c(k, A), e c(k, B))=e c(k, A)$ and $\max (\sec (k, A), \sec (k, B))=$ $\sec (k, A)$. Now from Axiom $2, \operatorname{ec}(k, A \wedge B)=e c(k, A)$ and $\sec (k, A \wedge B)=$ $\sec (k, A)$.

i.2.1) Let $q u a l(k, A)=0$ by Definition $2.2 \operatorname{ec}(k, A)=\sec (k, A)=1$ whence $e c(k, A \wedge B)=e c(k, A)$ and $\sec (k, A \wedge B)=\sec (k, A)$. Hence $e c(k, A \wedge$ $B)=\sec (k, A \wedge B)=1$ whence by Definition 2.2 qual $(k, A \wedge B)=0$. Hence qual $(k, A \wedge B)=\operatorname{qual}(k, A)$.

i.2.2) Let $q u a l(k, A)=1$ by Definition $2.2 \operatorname{ec}(k, A)=1$ and $\sec (k, A)=0$ whence $e c(k, A \wedge B)=e c(k, A)$ and $\sec (k, A \wedge B)=\sec (k, A)$. Whence $e c(k, A \wedge B)=1$ and $\sec (k, A \wedge B)=0$ and by Definition 2.2 qual $(k, A \wedge$ $B)=1$. Hence qual $(k, A \wedge B)=\operatorname{qual}(k, A)$.

i.2.3) Let $q u a l(k, A)=2$ by Definition 2.2 ec $(k, A)=0.5$ whence $e c(k, A \wedge B)=$ $e c(k, A)$. Thus $e c(k, A \wedge B)=0.5$ and by Definition 2.2 qual $(k, A \wedge B)=$ 2. Hence qual $(k, A \wedge B)=\operatorname{qual}(k, A)$.

i.2.4) Let $q u a l(k, A)=3$ by Definition $2.2 \operatorname{ec}(k, A)=0$ and $\operatorname{sef}(k, A)=0$. Dealing with these two cases in turn: $\left.a^{\prime}\right)$ As $e c(k, A \wedge B)=e c(k, A)$, $e c(k, A \wedge B)=0$. $\left.b^{\prime}\right)$ By Definition 2.1, min $(\operatorname{sef}(k, A), \operatorname{sef}(k, B))=0$; hence by Axiom $2 \operatorname{sef}(k, A \wedge B)=0$. From $\left.\left.a^{\prime}\right), b^{\prime}\right)$ and Definition 2.2, qual $(k, A \wedge B)=3$. Hence qual $(k, A B)=\operatorname{qual}(k, A)$. 
i.2.5) Let qual $(k, A)=4$ we have assumed at above that qual $(k, A) \leq$ qual $(k, B)$. By Definition 2.2, qual $(k, A)=\operatorname{qual}(k, B)=4$ also $e c(k, A)=0$ and $\operatorname{sef}(k, A)=\operatorname{sef}(k, B)=1$. Hence there are two cases: $\left.c^{\prime}\right) \operatorname{ec}(k, A \wedge B)=$ $e c(k, A), e c(k, A \wedge B)=0$ and $\left.d^{\prime}\right) \min (\operatorname{sef}(k, A), \operatorname{sef}(k, B))=1$ by Axiom $2 \operatorname{sef}(k, A \wedge B)=1$. By $\left.\left.c^{\prime}\right), d^{\prime}\right)$ and Definition 2.2, qual $(k, A \wedge B)=4$. Hence in this case also qual $(k, A \wedge B)=\operatorname{qual}(k, A)$. In $i .1)$ and $i .2)$, by assuming $q u a l(k, A)=\operatorname{qual}(k, B)$ we have shown qual $(k, A \wedge B)=$ $\min (q u a l(k, A), \operatorname{qual}(k, B))$.

Thus qual $(k, B) \leq$ qual $(k, A)$. Hence, qual $(k, A B)=$ $\min (q u a l(k, A), \operatorname{qual}(k, B))$.

The proof of Item $c$ ) is similar to the Item $b$ ).

Item $d$ ) using Proposition 3.8, items $a)$, c) we have qual $(k, A \wedge B)=$ qual $(k, A \wedge B)=\max (q u a l(k, A), \operatorname{qual}(k, B))=$ $\max (4-q u a l(k, A)$, qual $(k, B))$. By the Proposition 3.8, items $b), d)$ follow the last result.

\section{References}

[1] U. Aguero and S. Dasgupta, A Plausibility-Driven Approach to Computer Architecture Design, Comm. ACM, 30 (11) (1987), 922-932.

[2] U. Aguero, A Theory of Plausibility for Computer Architecture Designs, Dissertation Presented in the University of Southwestern Louisiana. In Partial Fulfilment of the Requirements for the Degree Doctor of Philosophy. (1987).

[3] A. Collins and R. Michalski, The logic of plausible reasoning: A core theory, Cognitive Science, 13 (1989), 1-49.

[4] S. Dasgupta and U. Aguero, On the Plausibility of Architectural Designs, Proc. 8th Int. Symp. on Computer Hardware Description Languages and their Applications, 87 (1987).

[5] A. R. Hooton, U. Aguero and S. Dasgupta, An Exercise in Plausibility Driven Design, IEEE Computer, 21 (7) (1988),21-31.

[6] P. A. Wilsey and S. Dasgupta, Functional and Operational Specifications of Computer Architectures,Proc. 9th Int. Conf. on Computer Hardware Description Languages and their Applications, 89 (1999).

\section{Received: September 30, 2006}

